\title{
Use of Pressure-Measuring Insoles to Characterize Gait Parameters in Simulated Reduced-Gravity Conditions
}

\author{
Christian Ison ${ }^{1, * \mathbb{D}}$, Connor Neilsen ${ }^{2}$, Jessica DeBerardinis ${ }^{2}$, Mohamed B. Trabia ${ }^{2} \mathbb{D}$ and Janet $S$. Dufek ${ }^{1}$ (D) \\ 1 Department of Kinesiology and Nutrition Sciences, University of Nevada, Las Vegas, NV 89154, USA; \\ janet.dufek@unlv.edu \\ 2 Department of Mechanical Engineering, University of Nevada, Las Vegas, NV 89154, USA; \\ connor.neilsen@colorado.edu (C.N.); deberardinis.jessica@gmail.com (J.D.); \\ mohamed.trabia@unlv.edu (M.B.T.) \\ * Correspondence: christian.ison@unlv.edu; Tel.: +1-626-824-4007
}

check for updates

Citation: Ison, C.; Neilsen, C.; DeBerardinis, J.; Trabia, M.B.; Dufek, J.S. Use of Pressure-Measuring Insoles to Characterize Gait Parameters in Simulated Reduced-Gravity Conditions. Sensors 2021, 21, 6244. https://doi.org/10.3390/s21186244

Academic Editor: Ajay Seth

Received: 28 July 2021

Accepted: 14 September 2021

Published: 17 September 2021

Publisher's Note: MDPI stays neutral with regard to jurisdictional claims in published maps and institutional affiliations.

Copyright: (c) 2021 by the authors. Licensee MDPI, Basel, Switzerland. This article is an open access article distributed under the terms and conditions of the Creative Commons Attribution (CC BY) license (https:/ / creativecommons.org/licenses/by/ $4.0 /)$.

\begin{abstract}
Prior researchers have observed the effect of simulated reduced-gravity exercise. However, the extent to which lower-body positive-pressure treadmill (LBPPT) walking alters kinematic gait characteristics is not well understood. The purpose of the study was to investigate the effect of LBPPT walking on selected gait parameters in simulated reduced-gravity conditions. Twenty-nine collegeaged volunteers participated in this cross-sectional study. Participants wore pressure-measuring insoles (Medilogic GmBH, Schönefeld, Germany) and completed three 3.5-min walking trials on the LBPPT (AlterG, Inc., Fremont, CA, USA) at 100\% (normal gravity) as well as reduced-gravity conditions of $40 \%$ and $20 \%$ body weight (BW). The resulting insole data were analyzed to calculate center of pressure (COP) variables: COP path length and width and stance time. The results showed that $100 \%$ BW condition was significantly different from both the $40 \%$ and $20 \%$ BW conditions, $p<0.05$. There were no significant differences observed between the $40 \%$ and $20 \%$ BW conditions for COP path length and width. Conversely, stance time significantly differed between the $40 \%$ and $20 \%$ BW conditions. The findings of this study may prove beneficial for clinicians as they develop rehabilitation strategies to effectively unload the individual's body weight to perform safe exercises.
\end{abstract}

Keywords: biomechanics; gait analysis; gait characteristics; pressure-measuring insoles; anti-gravity treadmill; reduced-gravity exercise; center of pressure; stance time

\section{Introduction}

Simulated reduced-gravity exercise is widely used for rehabilitating patients and athletes with the intention to facilitate early mobility while simultaneously adhering to safe aerobic exercise [1,2] and providing partial body weight support to perform simulated reduced-gravity exercise. The most common methods include the use of water immersion systems, also referred to as water or pool therapy, and harness suspension treadmill systems. Water immersion rehabilitation programs (pool therapy) incorporate movement when the body is partially submerged in water. Thus, a decrease in compressive forces on the musculoskeletal system due to the presence of buoyant forces acting in opposition to the body submerged in water is observed [3,4]. Another approach to unload partial body weight is to use a harness suspension treadmill system to vertically lift the individual partially off the ground [5]. A more recently developed method to provide partial body weight support during exercise is the use of a lower-body positive-pressure treadmill (LBPPT), which contains the lower body in an air-tight, increasingly pressurized air chamber to provide a lift force that effectively reduces musculoskeletal loading, i.e., body weight loading. The use of LBPPT is suggested to benefit those specifically recovering from lower extremity injuries and impairments. LBPPT exercise allows individuals and patients to perform safe aerobic exercises such as walking and running $[2,4]$. 
Prior reports have identified kinetic differences when comparing LBPPT walking with over-ground or normal-gravity condition walking. Researchers suggest that the clinical rehabilitative benefits for simulated reduced-gravity exercise include decreased ground reaction forces, increased lower extremity muscular strength, decreased lower extremity pain, and decreased overall exercise recovery time [6-9]. Although there is growing evidence that exercise performed in these reduced-gravity environments can be an alternative to exercise in normal gravity due to the capability of unloading a portion of body weight, the extent to which LBPPT walking alters kinematic gait characteristics is not well understood. It can be hypothesized that increasing the level of partial body weight support may potentially hinder gait stability due to changes in walking strategy and movement characteristics. Unstable gait leads to a compromised ability to control the position of the body's center of mass (COM) relative to the base of support (BOS) and is typically observed in individuals with neurological and musculoskeletal disorders [10,11]. Furthermore, recommendations of the optimal magnitude of partial body weight support that ensure non-alteration of gait were not studied [4,6,12-17]. In general, the effect of LBPPT on the potential deleterious gait characteristics has yet to be explored.

Center of pressure (COP) has been utilized as a means for assessing static and dynamic postural stability during gait analysis experiments [18,19]. COP, when examining kinetic plantar pressure measures, is typically used to identify and assess any issues with an individual's foot pathology during gait. The calculated COP location and its movement during the stance phase of each cycle is reported as an indicator of marked gait stability during dynamic tasks such as walking [20,21]. It was suggested that the assessment of plantar pressure could be an appropriate substitute for measuring foot trauma. In addition, COP path length and width have also been examined to determine preventative fall risk strategies, typically among geriatric and clinical populations [22,23]. Currently, pressure mats and force platforms are typically used to measure COP [19]. Recently, pressuremeasuring insoles have been suggested as an acceptable portable alternative to the existing gait analysis instruments [19]. Although pressure-measuring insoles typically sample at a lower frequency compared to force platforms, these frequencies are appropriate for measuring walking kinetics [24]. Pressure-measure insoles can also be used in various environments to record multiple consecutive steps, e.g., anti-gravity treadmill. Therefore, the purpose of our research was to investigate the effect of LBPPT walking on selected gait parameters in simulated reduced-gravity conditions. Specifically, we sought to explore changes in COP path length and width among normal and reduced body weight conditions to assess walking stability.

We hypothesized that (1) COP path length and width will change as gravity conditions are reduced, and (2) stance time measures will be identical across gravity conditions, as walking speed will be normalized for each participant performing the experiment. The findings from this study may provide additional information for clinicians and researchers in the rehabilitation and exercise prescription of LBPPT walking for those with impaired lower extremity function.

\section{Materials and Methods}

\subsection{Participants}

A convenience sample of 29 healthy, college-aged (24.7 \pm 4.0 years, 11 male, 18 female, mass $=72.4 \pm 13.5 \mathrm{~kg}$, height $=1.67 \pm 0.04 \mathrm{~m}$ ) (Table 1) volunteers was recruited for this experiment. Participants provided institutionally approved written consent (IRB \# 1510671-2) to participate. The inclusion criteria were (a) apparently healthy individuals between 18 and 55 years old, (b) no injuries or medical conditions that would limit the participant from being able to walk unsupported for $12 \mathrm{~min}$, (c) not pregnant or possibly pregnant, and (d) shoe size between 35 and 43 and not greater than 46 (European sizing), due to pressure-measuring insole size availability. 
Table 1. Demographic variables of participants.

\begin{tabular}{cccccc}
\hline $\begin{array}{c}\text { European } \\
\text { Insole Size }\end{array}$ & $\begin{array}{c}\text { Number of } \\
\text { Participants }\end{array}$ & Age (Year) & Mass (kg) & Height (m) & Gender \\
\hline $35-36$ & 5 & $22.8 \pm 3.96$ & $53.02 \pm 6.15$ & $1.53 \pm 0.04$ & Male (0) Female (5) \\
$37-38$ & 7 & $23.1 \pm 3.85$ & $56.49 \pm 8.39$ & $1.60 \pm 0.05$ & Male (0) Female (7) \\
$39-40$ & 5 & $26.2 \pm 2.59$ & $77.04 \pm 18.77$ & $1.65 \pm 0.04$ & Male (1) Female (4) \\
$41-42$ & 3 & $26.7 \pm 3.79$ & $79.5 \pm 18.19$ & $1.74 \pm 0.01$ & Male (1) Female (2) \\
$43-44$ & 4 & $24.5 \pm 5.00$ & $76.45 \pm 15.49$ & $1.69 \pm 0.06$ & Male (4) Female (0) \\
$45-46$ & 5 & $26 \pm 5.20$ & $91.68 \pm 14.15$ & $1.85 \pm 0.06$ & Male (5) Female (0) \\
\hline
\end{tabular}

\subsection{Instrumentation}

Two instruments were used in this experiment. The simulated reduced-gravity treadmill walking was performed using an AlterG Treadmill (AlterG, Inc., Fremont, CA, USA), Figure 1. The unweighting range of the AlterG is $100 \%$ to a minimum of $20 \%$ of the user's body weight. The speed ranges from 0 to $12 \mathrm{mph}$ [25].

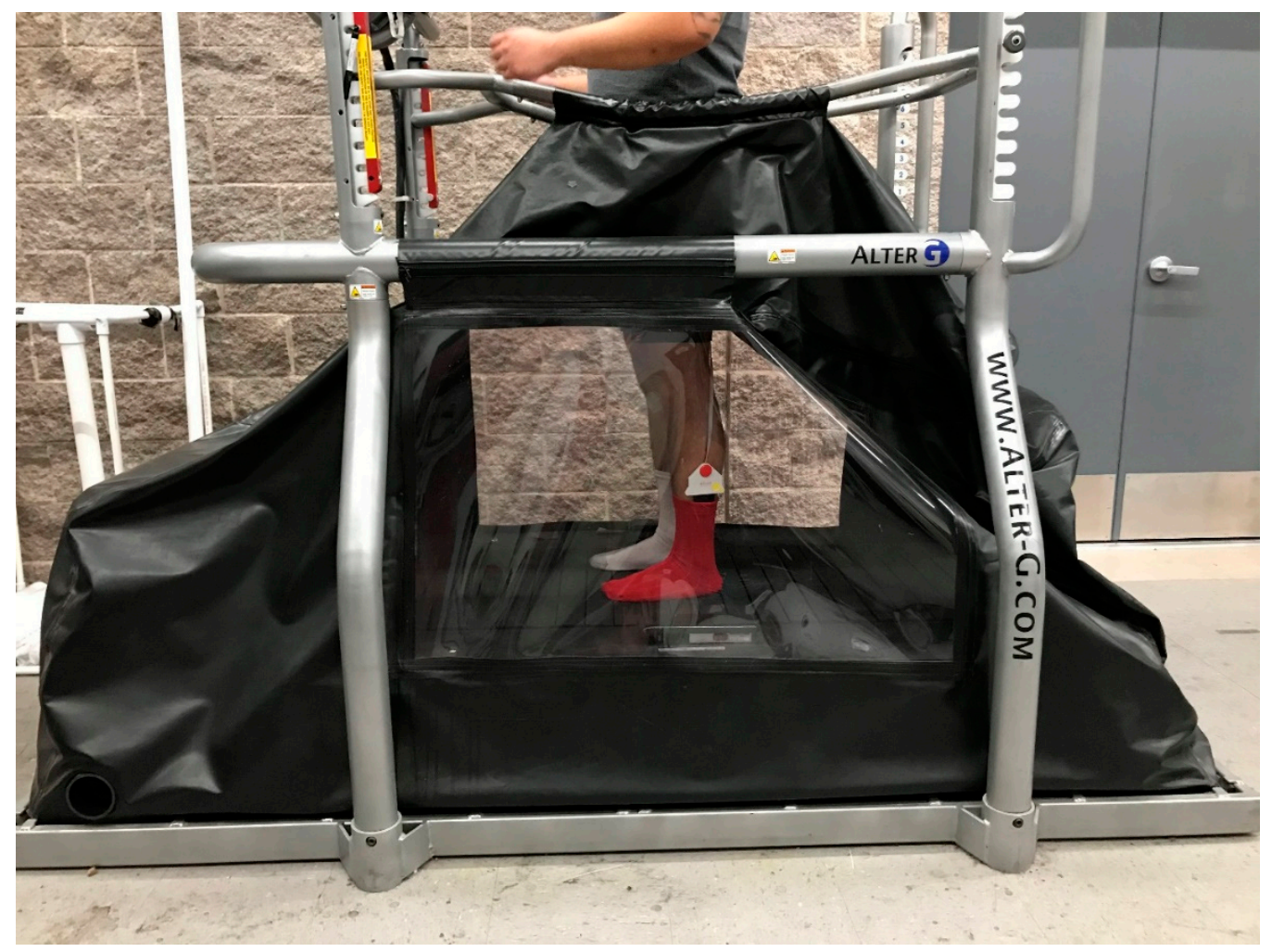

Figure 1. Subject using AlterG ${ }^{\circledR}$ Treadmill with Medilogic ${ }^{\circledR}$ Pressure-Measuring Insoles.

The second instrument was the Medilogic (Schönefeld, Germany, $60 \mathrm{~Hz}$ ) pressuremeasuring insole system, Figure 2. These insoles measure plantar pressure data using $1.50 \times 0.75 \mathrm{~cm}$ rectangular sensors that are arranged to match the plantar area. Six pressuremeasuring insole sizes were used in this experiment, with the number of sensors per insole ranging between 93 and 162 (Table 2). A wireless data modem, which was attached to the participant, sends the plantar pressure data from the modem to the computer [26]. 

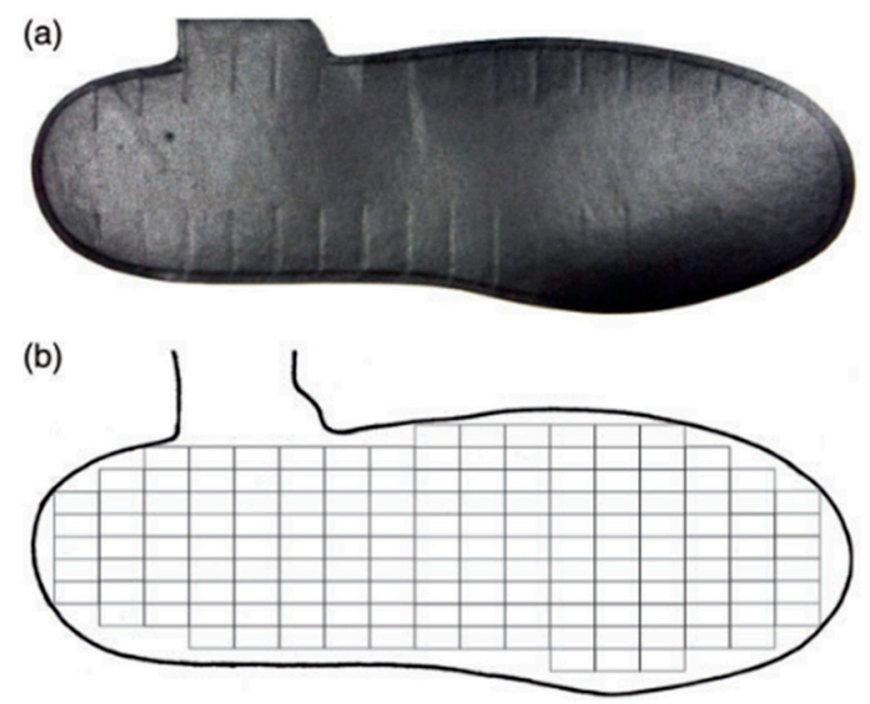

Figure 2. A Medilogic left insole, size 43-44 (a) and its corresponding sensor map where each rectangle represents a single sensor $(\mathbf{b})$.

Table 2. Number of sensors per insole.

\begin{tabular}{cc}
\hline Insole Size & Number of Sensors \\
\hline $35-36$ & 93 \\
$37-38$ & 107 \\
$39-40$ & 116 \\
$41-42$ & 130 \\
$43-44$ & 151 \\
$45-46$ & 162 \\
\hline
\end{tabular}

\subsection{Procedures}

Each participant was evaluated in a single laboratory visit. Following consent, anthropometric measures were obtained (shoe size, insole size, height, mass, gender, and age). Following the procedures of earlier researchers, participants placed their bare feet on appropriately sized Medilogic pressure-measuring insoles [19,27]. Insoles were taped to the feet to reduce the potential of insole slippage during the experiment. Participants were provided with socks that were worn over the insoles (Figure 1).

To eliminate noise resulting from the pressure generated by the interaction between feet, socks, and insoles, and to set a true zero pressure baseline, the following procedure was implemented at the start of each participant trial:

1. Seated participants were instructed to lift both feet approximately 1-2 inches above the floor.

2. Participants were instructed to stand quietly on the floor with weight equally distributed between the two limbs for a period of $15 \mathrm{~s}$.

3. Participants were instructed again to sit down and lift both feet approximately 1-2 inches above the ground.

The average bit value for both "feet in the air" phases was calculated. This value was labeled as zero bit (ZB).

Participants were then fitted with the AlterG neoprene compression shorts that include a kayak-style skirt that zips into the treadmill aperture to create an airtight seal near the participant's waist. The AlterG support bars were then aligned to the participant's iliac crest to ensure appropriate height (Figure 1). The treadmill was then manually calibrated based on the manufacturer's instructions. Participant-preferred walking speed was determined by setting the treadmill to $100 \%$ bodyweight (normal gravity) and the speed to a default of 2.5 miles per hour. Participants were asked to identify a self-selected comfortable walking 
speed by instructing the researcher on whether to increase or decrease the speed until the value was reached at which they would be walking comfortably for $15 \mathrm{~min}$. This speed was used for three gravity conditions defined as (1) $100 \%$ body weight or normal gravity, (2) $40 \%$ body weight, and (3) $20 \%$ body weight. Each walking condition lasted for $3.5 \mathrm{~min}$. Insole pressure data were recorded during the last $30 \mathrm{~s}$ of walking to represent the gait of each participant under the three different gravity conditions. The first 13 steps from each 30-s period were selected to be analyzed.

\subsection{Plantar Pressure Analysis}

Following the procedures of earlier researchers, insole data were processed to obtain the variables of interest for this study $[19,27]$. The specific dependent variables of interest were COP length (anterior-posterior excursion), COP width (medial-lateral excursion), and stance time.

Plantar pressure insole output data were recorded as $0-255$ bits. The digital output was converted into pressure according to the manufacturer's suggested conversion scale of 255 bits equal to $64 \mathrm{~N} / \mathrm{cm}^{2}$ using this formula:

$$
p_{i j}=D V_{i j} \frac{64 \mathrm{~N} / \mathrm{cm}^{2}}{255 \mathrm{bits}}
$$

where $D V_{i j}$ is the $0-255$ digital output of sensor $i$ at instant $j$.

$p_{i j}$ is the pressure of sensor $i$ at instant $j$.

The data were evaluated and processed using a MATLAB ${ }^{\circledR}$ (R2019a) custom code. The process started by using a low-pass fourth-order Butterworth filter with a cutoff frequency of $5 \mathrm{~Hz}$ to filter the data, which were then zeroed based on the average bit measurement of the noise cancellation phase. The cutoff frequency of $5 \mathrm{~Hz}$ was determined through previous experience with the insoles. This value was confirmed through inspection of the results of the data processing code. This filter was able to clearly identify the appropriate number of heel strikes and toe offs based on pressure data for all participants. Some isolated sensors had extremely low bit values due to the pressure of the socks. These sensors were eliminated using an adaptive threshold that identified active sensors in a step by including only sensors whose outputs were above the adaptive threshold of the most significant bit (MSB) instant [28]. This adaptive threshold technique was shown to be more effective in reducing plantar contact area error compared to the fixed-threshold technique.

The resulting bit data of all sensors were summed at each time instant and used to obtain the normal component of the ground reaction force. Sensors' readings are summed as

$$
F_{Z j}(N)=\sum_{i=1}^{n_{i}} p_{i j}\left(1.125 \mathrm{~cm}^{2}\right)
$$

where $F_{Z j}$ is the vertical component of the ground reaction force at instant $j$.

$n_{i}$ is the number of active sensors on insole $i$.

A $40 \mathrm{~N}$ normal force threshold was used to identify heel strike, toe off, and the overall number of steps within each condition [28].

Each sensor's location and coordinates for each insole size were calculated following the manufacturer's specifications by determining the $x$ and $y$ coordinates at the center of each active sensor based on the specific insole sensor map [19]. The active sensors were then used to calculate the coordinates of the COP using the following equation:

$$
\operatorname{COP}_{x, y_{j}}=\left(\frac{\sum_{i=1}^{n_{i}} p_{i j}\left(x_{i}\right)}{\sum_{i=1}^{n_{i}} p_{i j}}, \frac{\sum_{i=1}^{n_{i}} p_{i j}\left(y_{i}\right)}{\sum_{i=1}^{n_{i}} p_{i j}}\right)
$$

where $x i$ and $y i$ are the coordinates of sensor.

$\mathrm{COP}_{x, y_{j}}$ are the coordinates of COP at instant $j$. 
This phase of data reduction produced COP lengths during the first and last segments of the stance phase that were unrealistically long. This may have been a result of the stance thresholds not being based on the COP measurements but rather the ground reaction force values. Therefore, following the procedures of prior researchers, we examined segment lengths during the first or last quarters of the stance. COP length segments that were equal to or greater than $7 \mathrm{~mm}$ during the first or last quarter of the stance were identified and eliminated. It was observed that the first and last segment lengths of the stance were unrealistically long. As a result, exceeding tails and hooks that occur at toe off and heel strike may have been due to the resulting signal-to-noise ratio that typically occurs during locomotion. Visual inspections of this tail and hook were also seen from prior researchers in our laboratory. Therefore, we used this criteria threshold as established from prior studies [19]. Stance time measures were obtained as the time between peak force outputs. The average distance between peak force outputs was identified as stance time between each step. In order to compare COP among all participants, COP path length and width was normalized to the respective dimensions, length and width, of each insole. Left and right foot insole data were merged to a combined foot dataset.

\subsection{Statistical Analysis}

Recorded data were analyzed statistically using SPSS software (version 27.0). Two oneway repeated-measures ANOVAs with a Greenhouse-Geisser correction were performed to identify any significant differences between the dependent variables of COP path length and COP path width among each of the three gravitational conditions. Additionally, a oneway repeated-measures ANOVA was also performed to identify any significant differences in stance time measures among each of the three gravitational conditions. The level of statistical significance was set at $\alpha=0.05$.

\section{Results}

Table 3 presents the average and standard deviation values for the COP path length and width, normalized to insole size, for each of the three gravity conditions. ANOVA determined that average COP path length differed significantly among gravity conditions $(p<0.05)$. Post hoc tests using the Bonferroni correction revealed that the $100 \% \mathrm{BW}$ condition was significantly greater than both the $40 \%$ and $20 \%$ BW conditions. However, there were no significant differences observed between the 40\% BW and the $20 \%$ BW conditions $(p=0.259)$. A similar approach was used for COP width. Similar to COP length, ANOVA showed that the average COP path width differed significantly among gravity conditions $(p<0.05)$. Post hoc tests using the Bonferroni correction revealed that the $100 \%$ BW condition was significantly less than the $40 \%$ and $20 \%$ BW conditions. However, there were no significant differences observed between the $40 \%$ BW and $20 \%$ BW conditions $(p=0.134)$.

Table 3. Normalized average and standard deviation COP path length, COP path width, and stance time across each gravity condition.

\begin{tabular}{cccc}
\hline Gravity Condition & COP Path Length & COP Path Width & Stance Time \\
\hline $100 \%$ BW & $0.626 \pm 0.076$ & $0.109 \pm 0.023$ & $0.777 \pm 0.071$ \\
$40 \%$ BW & $0.438 \pm 0.223^{1}$ & $0.152 \pm 0.037^{1}$ & $0.753 \pm 0.096^{1}$ \\
$20 \%$ BW & $0.383 \pm 0.190^{1}$ & $0.162 \pm 0.043^{1}$ & $0.703 \pm 0.126^{1,2}$ \\
\hline
\end{tabular}

${ }^{1}$ Significantly different $(p<0.05)$ from $100 \%$ BW. ${ }^{2}$ Significantly different $(p<0.05)$ from $40 \%$ BW.

Table 3 presents the average and standard deviation values for the stance time measures, normalized to insole size, for each of the three gravity conditions. A repeatedmeasures ANOVA with a Greenhouse-Geisser correction determined that average stance differed significantly among gravity conditions $(p<0.05)$. Post hoc tests using the Bonferroni correction revealed that the $100 \% \mathrm{BW}$ condition was significantly greater than both 
the $40 \%$ and $20 \%$ BW conditions $(p<0.05)$. In addition, the $40 \% \mathrm{BW}$ condition was also statistically different from the $20 \%$ BW condition $(p<0.05)$.

\section{Discussion}

In this study, we found that the effect of lower-body positive-pressure treadmill (LBPPT) walking, specifically an increase in body weight support, or a reduction in gravity from $100 \%$ (normal gravity) to $40 \%$ and $20 \%$ (reduced gravity) body weight (BW), is mainly characterized by a significant decrease in center of pressure (COP) anterior-posterior segment length and simultaneous significant increase in COP medial-lateral segment width. The calculated COP location and its movement during the stance phase of each cycle are typically reported as indicators of marked gait stability during dynamic tasks such as walking $[20,21]$. This alteration in gait stability may be suggestive of a shift in walking patterns as gravity conditions are reduced. The decreasing anterior-posterior length may be suggestive of a shift from a rearfoot strike pattern toward a midfoot or forefoot strike pattern during LBPPT walking. Furthermore, the increasing medial-lateral COP width observed may be indicative of impaired gait stability, as the ability to stabilize the body's center of mass (COM) over its base of support (BOS) appears to be compromised during LBPPT walking, i.e., an attempt to establish medial-lateral directional stability [29].

Center of pressure (COP) measurements have been used by researchers and clinicians to assess static and dynamic postural stability impairments, as in individuals with diabetic peripheral neuropathy or Parkinson's disease [30,31]. Prior reports have suggested a decrease in COP path length and an increase in COP path width to be predictors of marked instability $[18,21,32]$. Furthermore, researchers have reported a change in COP during reduced-gravity exercise when compared with normal-gravity exercise [5,33-36].

It is important to note that the decrease in overall dynamic stability as gravity is reduced may be useful for clinicians and researchers, as this change in dynamic stability has been suggested as a possible indicator for fall risk, muscle weakness, and potential gait impairments. In addition, dynamic stability may provide an additional measure to determine when an individual or patient is able to further progress in the rehabilitation process. Therefore, the dynamic stability parameters evaluated in this study may provide important and valuable clinical insight.

Prior reports have demonstrated a reduction in the BOS to significantly increase COP displacement [10]. Although researchers have identified LBPPT walking as a potentially beneficial alternative for rehabilitation purposes due to its ability to reduce musculoskeletal load to recovering tissues, joints, and other biological structures, our findings suggest LBPPT walking may pose a potential compromise to gait stability [8,11]. Perhaps clinicians should advise against LBPPT rehabilitation programs whose primary focus is to rehabilitate postural control and stability impairments. It is suggested that LBPPT may be most clinically useful for those aiming to effectively reduce musculoskeletal load and to facilitate early mobility while simultaneously adhering to safe aerobic exercise.

The duration of the stance phase during each gait cycle (stance time) was obtained, and a statistically significant difference was found among each of the three gravity conditions. This was particularly interesting, as walking speed was standardized across gravity conditions for each participant, suggesting that stance times should be identical with no concomitant changes in walking kinematics. Because stance time decreased while velocity remained constant, it is feasible to suggest that with reduced gravity, stride rate increased. An increase in stride rate, given a constant velocity, could also suggest a decrease in stride length, as both (stride rate and length) inversely describe walking velocity. These gait characteristics, including step width and cadence, along with gait kinematics, e.g., trunk flexion, hip extension, knee flexion, and ankle dorsiflexion, are commonly evaluated by clinicians and rehabilitation specialists when examining gait abnormalities [13,37]. LBPPT walking may provide a viable instrument for those seeking to adjust or improve specific gait characteristics. The mean difference in stance times during the stance phase could also be indicative of a substantial alteration in gait stability in reduced-gravity environments. 
We speculate that the effect of LBPPT resulted in a more forefoot or "toe-walking" walking pattern, therefore reinforcing our postulation of a shift in walking strategy. On the contrary, a reduction in stance times would theoretically result in a reduction in compressive forces and ground reaction forces (GRF) exerted by the system, ultimately reducing musculoskeletal load. Hodges-Long et al. [12] found decreased plantar pressure measures during LBPPT running. However, future studies need to examine the degree to which reduced stance times correspond with reductions in GRF during LBPPT walking.

The results of the present study may be useful for clinicians and rehabilitation specialists in the programming of LBPPT exercise. Our results indicate that, at reduced gravity $(40 \%$ and $20 \% \mathrm{BW})$, an increase in instability is observed, as denoted by the increase in COP path width. Therefore, our findings suggest a potential threshold to which clinicians and specialists should perhaps prescribe LBPPT BW levels to the recovering individuals and patients. Future work is necessary to further confirm our findings and provide additional findings to further elucidate this potential threshold of gravity conditions.

Various limitations should be considered when interpreting the results of this study. Although plantar pressure data were recorded during the last $30 \mathrm{~s}$ of each walking condition, participants were not provided an accommodation time or learning phase for each of the gravitational walking conditions beyond the initial $2.5 \mathrm{~min}$ of walking prior to data collection. Variability in plantar pressure measures may have resulted from a lack of familiarity with LBPPT walking. Variability may have also occurred due to the relatively small sample size, perhaps resulting in type II error. In addition, the order in which participants performed the three walking trials, i.e., starting at $100 \%$ (normal gravity) BW, then $40 \%$, and finally $20 \%$, may have resulted in participant muscle fatigue. This fatigue may have contributed to the increased instability, as indicated by the decrease in COP path width. Lastly, we did not examine other gait parameters, such as stride length, step length, step width, etc., given the physical space limitations of the treadmill housing the pressurized air chamber combined with the specific focus of the present study, which was dynamic stability during reduced-gravity locomotion.

\section{Conclusions}

The purpose of the study was to investigate the effect of LBPPT walking on selected gait parameters in simulated reduced-gravity conditions. Our results indicated that as gravity was reduced, COP length increased and COP width decreased, while stance time decreased with a reduction in gravity. As well, the pattern of gait migrated for many participants for what is observed normally to be a toe-walking form of ambulation.

Future research should consider the use of a familiarization or accommodation period with respect to LBPPT walking. In addition, a larger sample size to strengthen the effect of plantar pressure measures performed in reduced-gravity locomotion may provide greater insight into LBPPT walking. Additionally, thresholds of reduced gravity more closely mimicking full body weight (e.g., $80 \%, 60 \%$ ) should be investigated to identify a more clinically feasible level of body weight reduction to allow for appropriate lower extremity loading while avoiding a migration to a toe-walking pattern of gait.

Author Contributions: Conceptualization, C.I., M.B.T. and J.S.D.; Data curation, C.I., C.N., J.D., M.B.T. and J.S.D.; Formal analysis, C.I., M.B.T. and J.S.D.; Investigation, C.N. and J.D.; Methodology, C.N. and J.D.; Project administration, M.B.T. and J.S.D.; Resources, M.B.T. and J.S.D.; Software, C.I., C.N., J.D., M.B.T. and J.S.D.; Supervision, C.I., J.D., M.B.T. and J.S.D.; Validation, C.I., M.B.T. and J.S.D.; Visualization, C.I., M.B.T. and J.S.D.; Writing—original draft, C.I.; Writing—review and editing, C.I., M.B.T. and J.S.D. All authors have read and agreed to the published version of the manuscript.

Funding: This research was partially supported by the Nevada NASA Space Grant Consortium (NVSGC).

Institutional Review Board Statement: The study was conducted according to the guidelines of the Declaration of Helsinki and approved by the Institutional Review Board of UNLV Biomedical IRB, Las Vegas, NV (\#1510671-2, May 2020). 
Informed Consent Statement: Informed consent was obtained from all subjects involved in the study.

Acknowledgments: Thank you to all the volunteers who participated in the study.

Conflicts of Interest: The authors declare no conflict of interest.

\section{References}

1. Kohzuki, M.; Miura, M.; Ito, O.; Nagasaka, M. Acute and Chronic Effects of Lower Body Positive Pressure Exercise on the Very Elderly: A Pilot Study. Int. J. Phys. Med. Rehabil. 2013, 1, 1-5. [CrossRef]

2. Lazaro, R. Effects of Lower Body Positive Pressure Treadmill Training on Balance, Mobility and Lower Extremity Strength of Community-Dwelling Older Adults: A Pilot Study. J. Allied Health 2020, 49, e99-e103. [PubMed]

3. Donelan, J.M.; Kram, R. The effect of reduced gravity on the kinematics of human walking: A test of the dynamic similarity hypothesis for locomotion. J. Exp. Biol. 1997, 200, 3193-3201. [CrossRef]

4. Saxena, A.; Granot, A. Use of an Anti-gravity Treadmill in the Rehabilitation of the Operated Achilles Tendon: A Pilot Study. J. Foot Ankle Surg. 2011, 50, 558-561. [CrossRef]

5. De Witt, J.K.; Perusek, G.P.; Lewandowski, B.E.; Gilkey, K.M.; Savina, M.C.; Samorezov, S.; Edwards, W.B. Locomotion in simulated and real microgravity: Horizontal suspension vs. parabolic flight. Aviat. Space Environ. Med. 2010, 81, 1092-1099. [CrossRef]

6. Kawae, T.; Mikami, Y.; Fukuhara, K.; Kimura, H.; Adachi, N. Anti-gravity treadmill can promote aerobic exercise for lower limb osteoarthritis patients. J. Phys. Ther. Sci. 2017, 29, 1444-1448. [CrossRef] [PubMed]

7. Peeler, J.; Takacs, J.; Leiter, J. Novel application of lower body positive-pressure in the rehabilitation of an individual with multiple lower extremity fractures. J. Rehabil. Med. 2011, 43, 653-656. [CrossRef] [PubMed]

8. Raffalt, P.C.; Hovgaard-Hansen, L.; Jensen, B.R. Running on a Lower-Body Positive Pressure Treadmill: VO2max, Respiratory Response, and Vertical Ground Reaction Force. Res. Q. Exerc. Sport 2013, 84, 213-222. [CrossRef] [PubMed]

9. Patil, S.; Steklov, N.; Bugbee, W.D.; Goldberg, T.; Colwell, C.W.; D’Lima, D.D. Anti-gravity treadmills are effective in reducing knee forces. J. Orthop. Res. 2012, 31, 672-679. [CrossRef] [PubMed]

10. Albertsen, I.M.; Ghédira, M.; Gracies, J.-M.; Hutin, É. Postural stability in young healthy subjects-Impact of reduced base of support, visual deprivation, dual tasking. J. Electromyogr. Kinesiol. 2017, 33, 27-33. [CrossRef]

11. Kristiansen, M.; Odderskær, N.; Kristensen, D.H. Effect of body weight support on muscle activation during walking on a lower body positive pressure treadmill. J. Electromyogr. Kinesiol. 2019, 48, 9-16. [CrossRef]

12. Long, L.H.-; Cross, K.; Magrum, E.; Feger, M.; Hertel, J. The effect of body weight reduction using a lower body positive pressure treadmill on plantar pressure measures while running. Phys. Ther. Sport 2020, 43, 100-107. [CrossRef] [PubMed]

13. Barnes, K.R.; Janecke, J.N. Physiological and Biomechanical Responses of Highly Trained Distance Runners to Lower-Body Positive Pressure Treadmill Running. Sports Med. Open 2017, 3, 41. [CrossRef]

14. Everett, M.E.; O'Connor, D.P.; DeWitt, J.K. Lower limb position during treadmill jogging and fast running in microgravity. Aviat. Space Environ. Med. 2009, 80, 882-886. [CrossRef] [PubMed]

15. Gosseye, T.P.; Willems, P.A.; Heglund, N.C. Biomechanical analysis of running in weightlessness on a treadmill equipped with a subject loading system. Graefe's Arch. Clin. Exp. Ophthalmol. 2010, 110, 709-728. [CrossRef] [PubMed]

16. Schaffner, G.; Dewitt, J.; Bentley, J.R.; Yarmanova, E.; Kozlovskaya, I.B.; Hagan, D. Effect of Load Levels of Subject Loading Device on Gait, Ground Reaction Force, and Kinematics during Human Treadmill Locomotion in a Weightless Environment; National Aeronautics and Space Administration, Johnson Space Center: Houston, TX, USA, 2005.

17. Sylos-Labini, F.; Ivanenko, Y.P.; Cappellini, G.; Portone, A.; MacLellan, M.J.; Lacquaniti, F. Changes of Gait Kinematics in Different Simulators of Reduced Gravity. J. Mot. Behav. 2013, 45, 495-505. [CrossRef]

18. Li, B.; Xiang, Q.; Zhang, X. The center of pressure progression characterizes the dynamic function of high-arched feet during walking. J. Leather Sci. Eng. 2020, 2, 1-10. [CrossRef]

19. DeBerardinis, J.; Neilsen, C.; E Lidstone, D.; Dufek, J.S.; Trabia, M.B. A comparison of two techniques for center of pressure measurements. J. Rehabil. Assist. Technol. Eng. 2020, 7, 205566832092106. [CrossRef]

20. Xiu, W.; Ruble, K.; Ma, O. A reduced-gravity simulator for physically simulating human walking in microgravity or reducedgravity environment. In Proceedings of the 2014 IEEE International Conference on Robotics and Automation (ICRA), IEEE, Hong Kong, China, 31 May-7 June 2014; pp. 4837-4843.

21. Lemay, J.-F.; Gagnon, D.H.; Nadeau, S.; Grangeon, M.; Gauthier, C.; Duclos, C. Center-of-pressure total trajectory length is a complementary measure to maximum excursion to better differentiate multidirectional standing limits of stability between individuals with incomplete spinal cord injury and able-bodied individuals. J. Neuroeng. Rehabil. 2014, 11, 8. [CrossRef] [PubMed]

22. Tahir, A.M.; Chowdhury, M.E.H.; Khandakar, A.; Al-Hamouz, S.; Abdalla, M.; Awadallah, S.; Reaz, M.B.I.; Al-Emadi, N. A Systematic Approach to the Design and Characterization of a Smart Insole for Detecting Vertical Ground Reaction Force (vGRF) in Gait Analysis. Sensors 2020, 20, 957. [CrossRef]

23. Villa-Parra, A.C.; Lima, J.; Delisle-Rodriguez, D.; Vargas-Valencia, L.; Neto, A.F.; Bastos, T. Assessment of an Assistive Control Approach Applied in an Active Knee Orthosis Plus Walker for Post-Stroke Gait Rehabilitation. Sensors 2020, 20, 2452. [CrossRef]

24. Winter, D.A. Camera speeds for normal and pathological gait analyses. Med. Biol. Eng. Comput. 1982, 20, 408-412. [CrossRef] 
25. Frequently Asked Questions-Service. AlterG. Available online: https://www.alterg.com/service-faq (accessed on 9 October 2020).

26. Noraxon, Medilogic Wireless Foot Pressure Measurement System. Available online: https://www.noraxon.com/products/ pressure-and-force-measurement-technology / medilogic-insoles / (accessed on 25 September 2017).

27. DeBerardinis, J.; Dufek, J.S.; Trabia, M.B.; E Lidstone, D. Assessing the validity of pressure-measuring insoles in quantifying gait variables. J. Rehabil. Assist. Technol. Eng. 2018, 5, 2055668317752088. [CrossRef] [PubMed]

28. Lidstone, D.E.; DeBerardinis, J.; Dufek, J.S.; Trabia, M.B. Electronic measurement of plantar contact area during walking using an adaptive thresholding method for Medilogic ${ }^{\circledR}$ pressure-measuring insoles. Foot 2019, 39, 1-10. [CrossRef] [PubMed]

29. Ku, P.; Abu Osman, N.; Abas, W.W. The limits of stability and muscle activity in middle-aged adults during static and dynamic stance. J. Biomech. 2016, 49, 3943-3948. [CrossRef] [PubMed]

30. Schmit, J.M.; Riley, M.A.; Dalvi, A.; Sahay, A.; Shear, P.K.; Shockley, K.D.; Pun, R.Y.K. Deterministic center of pressure patterns characterize postural instability in Parkinson's disease. Exp. Brain Res. 2005, 168, 357-367. [CrossRef]

31. Dixit, S.; Maiya, G.A. Diabetic peripheral neuropathy and its evaluation in a clinical scenario: A review. J. Postgrad. Med. 2014, 60, 33-40. [CrossRef]

32. Hu, X.; Zhao, J.; Peng, D.; Sun, Z.; Qu, X. Estimation of Foot Plantar Center of Pressure Trajectories with Low-Cost Instrumented Insoles Using an Individual-Specific Nonlinear Model. Sensors 2018, 18, 421. [CrossRef]

33. De Witt, J.K.; Ploutz-Snyder, L.L. Ground reaction forces during treadmill running in microgravity. J. Biomech. 2014, 47, 2339-2347. [CrossRef]

34. De Witt, J.K.; Schaffner, G.; Ploutz-Snyder, L.L. Bungee force level, stiffness, and variation during treadmill locomotion in simulated microgravity. Aviat. Space Environ. Med. 2014, 85, 449-455. [CrossRef]

35. Li, X.; Aruin, A.S. Anticipatory postural adjustments in conditions of simulated reduced gravity. Gait Posture 2008, 28, 538-544. [CrossRef] [PubMed]

36. Labini, F.S.; Lacquaniti, F.; Ivanenko, Y.P. Human Locomotion under Reduced Gravity Conditions: Biomechanical and Neurophysiological Considerations. BioMed Res. Int. 2014, 2014, 1-12. [CrossRef] [PubMed]

37. Wert, D.M.; Brach, J.; Perera, S.; Vanswearingen, J.M. Gait Biomechanics, Spatial and Temporal Characteristics, and the Energy Cost of Walking in Older Adults with Impaired Mobility. Phys. Ther. 2010, 90, 977-985. [CrossRef] [PubMed] 